\title{
Etude Préliminaire de la Diversité et de L'abondance des Coléoptères Coprophages du Parc National de Taï, Sud-Ouest Côte d'Ivoire
}

\section{Célestin Yao Kouakou, Maître-Assistant}

Université Jean Lorougnon Guédé,

Laboratoire de Biodiversité et Ecologie Tropicale, Côte d'Ivoire

Ossey Robert N'Depo, Maître-Assistant

Senan Soro, Maître de Conférences

Sékongo Kolo, Doctorant

Université Jean Lorougnon Guédé,

Laboratoire d'Amélioration de la Protection Agricole, Côte d'Ivoire

Doi:10.19044/esj.2019.v15n36p45 ～URL:http://dx.doi.org/10.19044/esj.2019.v15n36p45

\section{Résumé}

Les insectes coprophages exploitent les crottes des mammifères, et contribuent, ainsi, à l'entretien et à l'équilibre des écosystèmes. Cependant, les études sur l'entomofaune des crottes de mammifères restent rares au Parc National de Taï (PNT), Sud-Ouest Côte d'Ivoire. Ce travail vise à identifier les Coléoptères coprophages inféodés aux crottes de grands mammifères du PNT, pour contribuer à une meilleure connaissance de l'entomofaune de l'aire protégée. Les crottes de mammifères détectées étaient identifiées, prélevées, puis transportées jusqu'à une source d'eau proche. Chaque échantillon de crottes étiqueté est mis dans un seau d'eau dans lequel les insectes sont récoltés par flottaison, puis conservés. Au total, 226 Coléoptères Scarabaeidae appartenant à deux Sous-familles (Coprinae et Scarabeinae) et quatre Tribus (Aphodiini, Canthonini, Onthophagini et Sysiphini) ont été observés. Les Onthophagini, qui sont de la guilde des fouisseurs, étaient les plus abondants et représentaient $66,37 \%$ des individus collectés. Les Sysiphini (rouleurs) étaient les moins abondants et représentaient $4,42 \%$ des individus observés. Une différence significative a été observée entre l'abondance de Coléoptères coprophages des crottes de l'éléphant et celle des crottes d'autres mammifères notamment le potamochère $(\mathrm{p}=0,03)$, l'hippopotame nain $(\mathrm{p}=$ $0,04)$, et céphalophes $(\mathrm{p}=0,03)$. Cependant, aucune différence significative n'a été observée entre l'abondance de Coléoptères coprophages des crottes de l'éléphant et de celles du buffle $(p=0,06)$. Cette étude mérite d'être approfondie pour mieux cerner les relations entre les divers taxa et pour le 
suivi adéquat de la faune qui est nécessaire pour la gestion et la conservation du PNT.

Mots clés: Coléoptères coprophages, Crotte, Mammifères, Parc National de Taï

\title{
Preliminary Study of the Diversity and Abundance of Dung Beetles in Taï National Park, South-West Côte d'Ivoire
}

\section{Célestin Yao Kouakou, Maître-Assistant}

Université Jean Lorougnon Guédé,

Laboratoire de Biodiversité et Ecologie Tropicale, Côte d'Ivoire

Ossey Robert N'Depo, Maître-Assistant

Senan Soro, Maître de Conférences

Sékongo Kolo, Doctorant

Université Jean Lorougnon Guédé,

Laboratoire d'Amélioration de la Protection Agricole, Côte d'Ivoire

\begin{abstract}
Coprophagous insects exploit dung piles of mammals, thus they contribute, to ecosystem maintenance and balance. However, studies on the entomofauna of mammals' dung remain rare at Taï National Park (TNP), Southwestern Côte d'Ivoire. This study aims at identifying coprophagous beetles that feed on large mammals' dung at TNP to contribute to a better knowledge of the entomofauna of the protected area. Mammals dung piles that were detected, were identified, removed, and brought to a nearby source of water. Each dung sample was tagged and placed in a bucket containing water in which the dung beetles floundered and then kept in a vial. In total, 226 Scarabaeidae dung beetles belonging to two subfamilies (Coprinae and Scarabeinae) and four tribes (Aphodiini, Canthonini, Onthophagini and Sysiphini) were observed. The Onthophagini, which are from the burrowing guild, were the most abundant and represented $66.37 \%$ of the individuals observed. Sysiphini (rollers) were the least abundant and represented 4.42\% of the individuals observed. A significant difference was observed between the abundance of coprophagous beetles in the dung forest elephant and that of the dung of other mammals including the bush pig $(\mathrm{p}=0.03)$, the pygmy
\end{abstract}


hippopotamus $(\mathrm{p}=0.04)$, and duikers $(\mathrm{p}=0.03)$. However, there was no significative difference between the abundance of coprophagous beetle from forest elephant's and forest buffalo's dungs $(p=0.06)$. This study deserves further investigation to better understand the relationships between various taxa and for adequately monitoring the fauna and improve the management conservation of the protected area.

Keywords: Coprophagous beetles, Dung, Mammals, Taï National Park

\section{Introduction}

La connaissance de la diversité biologique des milieux forestiers est indispensable à l'établissement de bases satisfaisantes pour la gestion durable et la conservation en raison des taux élevés de déforestation et de la perte massive des espèces (Couturier \& Guillaumet, 1997). La zone forestière de la Côte d'Ivoire fait partie intégrante de la forêt de la haute Guinée, une zone de grande importance eu égard à sa grande diversité floristique et faunique (Myers et al., 2000 ; Kolongo et al., 2006). Malheureusement, cette zone subit une pression anthropique. La compréhension des relations entre les composantes de la diversité biologique de ces milieux forestiers semble nécessaire pour évaluer et prévoir les conséquences environnementales réelles des activités humaines (Kouakou, 2004 ; Nichols et al., 2008, Zinga et al., 2016).

Composantes abondantes des écosystèmes, les insectes et les mammifères sont des acteurs clés de la majorité des processus écosystémiques. En milieu tropical, les mammifères sont particulièrement menacées d'extinction du fait de la chasse pour la viande de brousse (Ripple et al., 2016). Pourtant, leur perte peut avoir des effets négatifs dans tout l'écosystème (Coleman \& Hendrix, 2000 ; Estes, 2016). En effet, de nombreuses espèces d'insectes vivent aux dépens des mammifères. C'est le cas de coléoptères coprophages ou bousiers qui pourraient être fortement affectés et plus rapidement que d'autres taxa par le changement des composantes du milieu (Samways, 1993). La vie ou le développement de ces insectes est lié aux excréments d'autres animaux essentiellement mammifères (Cambefort, 1980). La consommation, l'enfouissement et l'aération des excréments par ces coléoptères permettent une remise en circulation des éléments minéraux, augmentant ainsi la productivité des écosystèmes et assurant une production fourragère de qualité (Simon, 2010). Les coléoptères coprophages sont observés majoritairement dans les pâturages et les milieux naturels où vit la faune mammalienne notamment les éléphants, les buffles, etc. (Dosso et al., 2018). Ces insectes constituent un excellent taxon focal pour comprendre des interactions entre les structures anthropogènes de perturbation et des communautés (Favila \& Halffter, 1997). La richesse spécifique de ce 
taxon, décrite comme la plus importante en Afrique avec plus de 2120 espèces, serait liée à l'abondance en mammifères (Hanksi \& Cambefort, 1991 ; Zinque, 2012).

Dernier bloc important de la forêt primaire de l'Afrique de l'Ouest, le Parc National de Taï (PNT) en Côte d'Ivoire est une réserve de biosphère et site du patrimoine mondial de l'UNESCO. Il revêt une importance fondamentale pour la connaissance de la faune entomologique forestière (Cambefort, 1985). Au PNT, les travaux sur des aspects de l'écologie des coléoptères bousiers datent de plus d'une décennie (Cambefort, 1985). Par ailleurs, le suivi de la faune mammalienne du PNT indique une baisse des populations au cours des dernières décennies (Hoppe-Dominik et al., 2011). Il est donc nécessaire de fournir des informations actualisées sur la diversité des coléoptères coprophages mais aussi d'investiguer la relation d'abondance entre ces insectes et des crottes spécifiques à des mammifères.

L'objectif général était de donner un aperçu du peuplement de coléoptères coprophages du PNT afin de contribuer à une meilleure connaissance de l'entomofaune en relation avec la présence de mammifères dans cette aire protégée. Les objectifs spécifiques de l'étude sont (1) déterminer la richesse taxonomique des coléoptères coprophages du PNT avec l'identification des Tribus de ces insectes ; (2) déterminer l'abondance des coléoptères coprophages associés aux crottes de différents mammifères notamment le buffle de forêt (Syncerus caffer nanus), l'éléphant de forêt (Loxodonta cyclotis), hippopotame nain (Choeropsis liberiensis), potamochère (Potamochoerus porcus), des céphalophes (Cephalophus sp.).

\section{Matériel et méthodes \\ Site d'étude}

L'étude a été réalisée au PNT qui est situé au Sud-Ouest de la Côte d'Ivoire, entre $5^{\circ} 08^{\prime}$ et $6^{\circ} 24^{\prime}$ de latitude Nord et $6^{\circ} 47^{\prime}$ et $7^{\circ} 25^{\prime}$ de longitude Ouest (OIPR, 2014). Le parc est soumis à un climat équatorial de transition à 4 saisons. Ce climat est caractérisé par 2 saisons de pluies : une grande (mars à juin) et une petite (septembre à novembre). Elles sont alternées par une grande saison sèche (décembre à février) et une petite saison sèche (juillet à août). La moyenne des précipitations annuelles est d'environ $1800 \mathrm{~mm}$ et la température moyenne mensuelle est de $24^{\circ} \mathrm{C}$ à $28^{\circ} \mathrm{C}$ (Chatelain et al., 2001). Le PNT abrite environ 145 espèces de mammifères, correspondant à $93 \%$ des espèces de mammifères de la zone forestière ouest-guinéenne (Riezebos et al., 1994). D'autres détails sur le climat, la faune et la flore du PNT sont fournis par Bousquet (1978) et Riezebos et al., (1994). 


\section{Collecte des données}

Les crottes de moyens et grands mammifères étaient recherchées en Octobre 2017 dans les secteurs Taï et Djouroutou du PNT par une équipe expérimentée en inventaire faune notamment dans l'identification des espèces de mammifères à partir de leurs indices de présence (crottes, empreintes, vocalisations etc.). En effet, depuis 2005, des membres de cette équipe participaient annuellement, pendant quatre à cinq mois, à la collecte de données d'un programme de suivi de la faune au PNT (Hoope-Dominik, 2011 ; N'goran, 2015). La recherche de crottes était faite en suivant des itinéraires prédéterminés pouvant toutefois dévier de l'axe défini (au maximum $40^{\circ}$ ) en utilisant, le cas échéant, un chemin de moindre résistance (White \& Edwards, 2000). La vitesse moyenne de marche de l'équipe pour rechercher les crottes étaient d'environ un kilomètre par heure. Pour les crottes d'une même espèce de mammifère qui sont reconnaissables par leur volume, les empreintes au sol, etc. (Bang \& Dahlstrom, 2001), la distance entre deux points successifs d'échantillonnage était de deux kilomètres pour couvrir une grande zone représentative des secteurs prospectés dans le parc, pendant la période d'étude.

Lorsqu'une crotte de mammifère était observée, l'équipe identifiait l'espèce animale et notait le type d'habitat (végétation, la proximité d'une rivière, etc.) dans lequel l'observation était faite. Les types d'habitat définies par Tiedoue et al., (2013) sont : Forêt dense Sous-Bois Ouvert (FSO), Forêt dense Sous-Bois Fermé (FSF), Forêt sur sols hydromorphes (FSHD), Forêt des inselbergs ou de montagne (FIMT), Forêt secondaire jeune ou Fourrés (FSJF) et Brousses ou Végétation non ligneuse dominée par des herbacées (BVNL). La crotte était prélevée avec le sol sous adjacent, à l'aide d'une pelle et une daba, puis mise dans des sachets portant le numéro de l'échantillon. La présence de sable à la surface de la crotte indique la présence de fouisseurs qui sont dénichés en utilisant d'une daba (Kouakou, 2004). Après le prélèvement, les sachets sont fermés hermétiquement et transportés jusqu'à une source d'eau proche. Chaque échantillon étiqueté était mis dans un seau contenant de l'eau, puis les coléoptères coprophages présents étaient récoltés dans le surnageant et conservés dans un flacon qui contenait de l'alcool à $70 \%$ et portait la même étiquette que l'échantillon. La recherche des crottes s'effectuait entre huit (8) heures du matin et 17 heures pour s'assurer d'une bonne visibilité dans la forêt dense du PNT. Dix jours de collecte de d'échantillons ont été nécessaires pour cette étude qui couvrait environ $250 \mathrm{~km}^{2}$.

\section{Identification des insectes et analyses statistiques des données}

Pour déterminer la diversité et l'abondance des coléoptères coprophages, les insectes collectés dans les crottes ont été identifiés aux 
niveaux famille, sous-famille et tribu puis le nombre d'individu a été compté. Les différents niveaux taxonomiques identifiés permettent d'apprécier la diversité de ces coléoptères. Les clés d'identification des coléoptères coprophages utilisées sont celles fournies par Cambefort \& Bordat (2003), Krell et al. (2003), Krell-Westerwalbesloh et al. (2004) puis Davis et al. (2008). Les insectes étaient observés sous une loupe binoculaire, ensuite par comparaison avec les caractères décrits dans les clés, la tribu à laquelle appartient chaque spécimen était déterminée. Après l'identification, les insectes ont été mis dans des petits bocaux de conservation contenant du papier hygiénique imbibée d'une solution à $70 \%$ d'alcool préparée à cet effet (Danon et al., 2017 ; Kouakou, 2004). Les bocaux de conservation étaient fermés hermétiquement puis étiquetés en marquant le numéro de l'échantillon, la date, l'heure et site d'échantillonnage.

L'abondance des individus de chaque tribu a été déterminée en faisant la somme du nombre d'individus de la tribu des différents échantillons. L'abondance relative (Ar) d'une tribu "i", exprimée en pourcentage, a été déterminée en utilisant la formule suivante $: \mathrm{Ar}=100 \mathrm{xn}_{\mathrm{i}} / \mathrm{N}$ où $\mathrm{n}_{\mathrm{i}}$ est le nombre d'individus de la tribu "i" prise en considération et $\mathrm{N}$ le nombre total des individus de toutes les tribus confondues (Zaime \& Gautier, 1989).

Pour comparer les moyennes d'abondance d'insectes coprophages dans les crottes de différents mammifères, des analyses de variances suivies du test d'homogénéité ou test de différence significative minimale (Test LSD de Fisher), au seuil de 5\%, ont été faites avec le logiciels $\mathrm{R}$ version 2.8.

\section{Résultats et discussion}

\section{Résultats}

\section{Richesse taxonomique et guildes de coléoptères coprophages}

$\mathrm{Au}$ total, 32 échantillons de crottes de mammifères comprenant 226 individus de coléoptères coprophages ont été recensés dans deux types d'habitat : FSO et FSDH.

Le Tableau 1 indique que les insectes observés appartiennent à la famille des Scarabaeidae et sont repartis en trois sous-familles (Aphodiinae, Coprinae et Scarabaeinae) et quatre tribus (Aphodiini, Canthonini, Onthophagini et Sysiphini). Une image d'un représentant de chaque tribu est présenté dans le support complémentaire 1 (Sup.C1) Ils comprenaient trois guildes fonctionnelles dont des rouleurs représentés par les Sysiphini et les Canthonini, des fouisseurs représentés par les Onthophagini et des résidents représentés par des Aphodiini. 
Tableau 1 : Richesse taxonomique des Coléoptères coprophages et leurs abondances observées dans les crottes de Mammifères au PNT

\begin{tabular}{ccccc}
\hline Famille & Sous-famille & Tribu & Guilde & Abondance individus \\
\hline \multirow{2}{*}{ Scarabaeidae } & Coprinae & Aphodiini & Résident & 60 \\
& & Canthonini & Rouleur & 6 \\
& Scarabaeinae & & & 150 \\
& & Onthophagini & Fouisseur & 10 \\
\hline Total & 02 & Sysiphini & Rouleur & 10 \\
\hline
\end{tabular}

\section{Abondance relative des Coléoptères coprophages dans les crottes de mammifères}

Dans l'ensemble, les Onthophagini étaient les plus abondants (150 individus) tandis les Canthonini étaient les moins abondants (six individus) dans les crottes de mammifères avec respectivement $66,37 \%$ et $2,65 \%$ des observations (Figure 1).

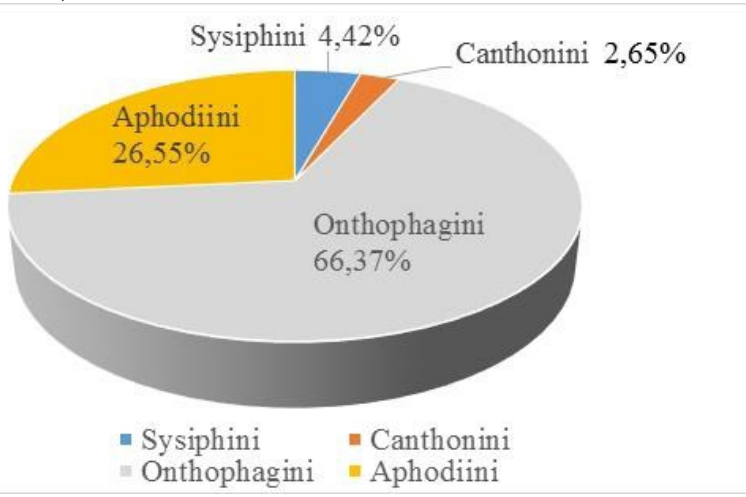

Figure 1 : Abondance relative des Tribus de Coléoptères coprophages au PNT.

Dans chacun des deux types d'habitat, les rouleurs (Sysiphini et Canthonini) représentaient moins de $10 \%$ des insectes observés. Les fouisseurs (Onthophagini) étaient les plus abondants aussi bien en FSO qu'en FSDH avec respectivement $62 \%$ et $78 \%$ des observations (Figure 2 ). 

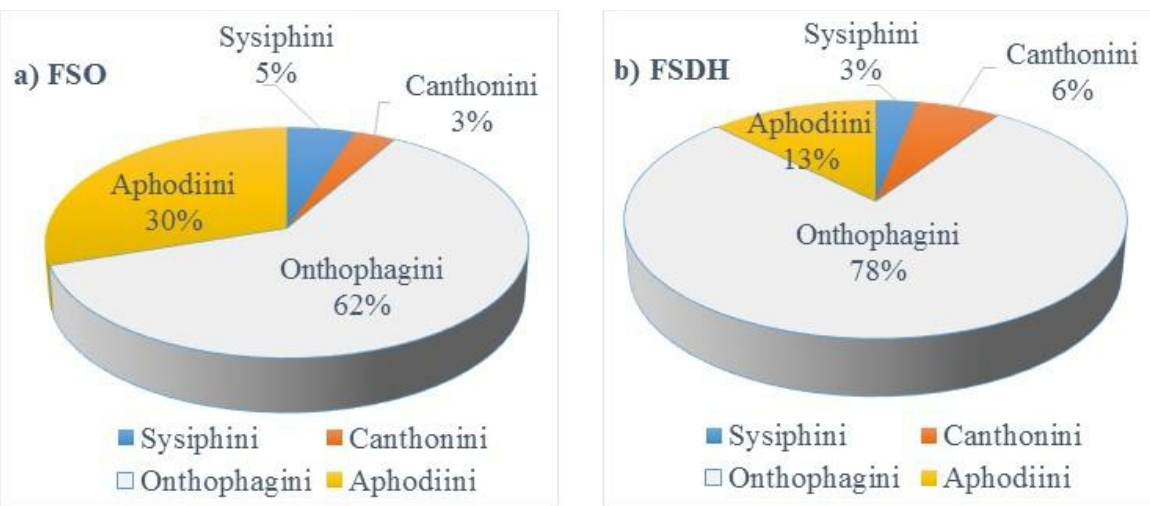

Figure 2 : Abondance relative des tribus de coléoptères coprophages en FSO (a) et en FSHD (b) au PNT

L'effectif moyen des coléoptères coprophages dans les crottes variait avec l'espèce de mammifère responsable de la crotte (Figure 3). En effet, ils étaient plus abondants dans les crottes de l'éléphant de forêt (Loxodonta cyclotis) avec une moyenne de 6,13 individus/crotte. Les crottes de l'hippopotame nain (Choeropsis liberiensis) renfermaient moins d'insectes coprophages avec une moyenne de 1,75 individus/crottes. Les crottes du buffle de forêt (Syncerus caffer nanus), des céphalophes (Cephalophus sp.) et du potamochère (Potamochoerus porcus) regorgeaient respectivement 4,71 individus/crotte, 3,22 individus/crotte, et 1,83 individus/crotte.

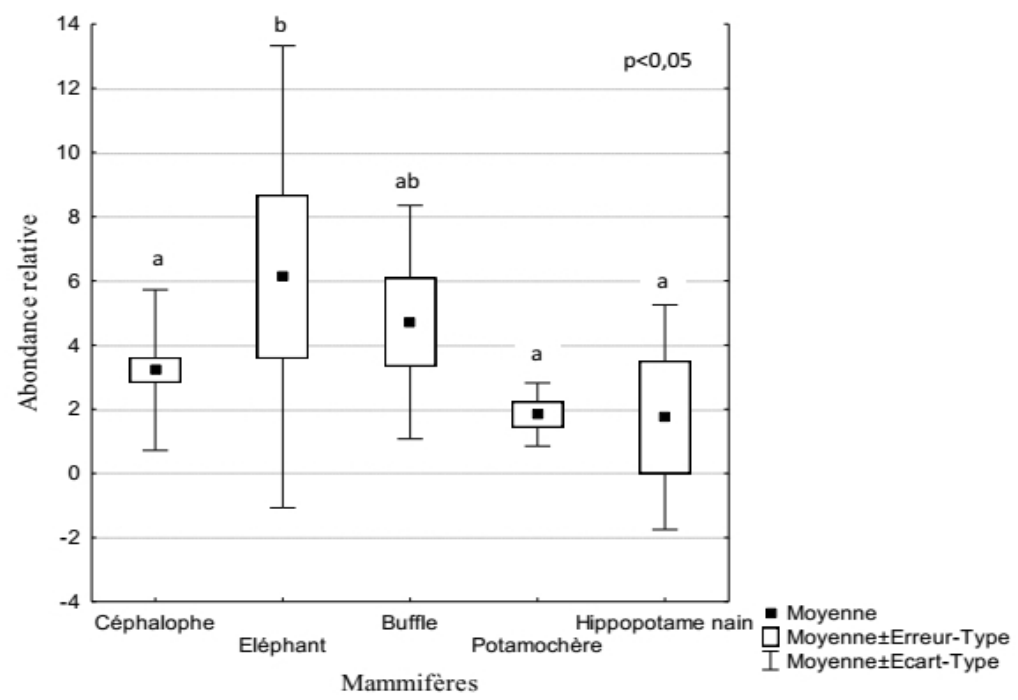

Figure 3 : Comparaison de l'abondance moyenne de coléoptères coprophages dans les crottes des mammifères. Les moyennes suivies de la même lettre sont significativement identiques (Test LSD de Fisher, a=0,05).

Une différence significative a été observée entre l'abondance moyenne de coléoptères coprophages dans les crottes l'éléphant de forêt et celle des 
crottes de céphalophes $(\mathrm{p}=0,03)$. Une différence similaire a été observée au niveau de l'éléphant de forêt et de potamochère $(\mathrm{p}=0,03)$; puis au niveau l'éléphant de forêt et de l'hippopotame nain $(\mathrm{p}=0,04)$ (Figure 3). Par contre, il n'y avait pas de différence significative entre l'effectif moyen de coléoptères coprophages dans les crottes de l'hippopotame nain et dans celles du buffle ( $p$ $=0,05)$, puis entre l'effectif moyen de coléoptères coprophages dans les crottes de l'éléphant de forêt et dans celles du buffle de forêt $(p=0,06)$.

\section{Discussion}

La connaissance de l'entomofaune inféodée aux crottes de mammifères est importante dans la compréhension des relations entre deux composantes essentielles d'un écosystème tropical. Au cours de ce travail réalisé au PNT, la richesse taxonomique des coléoptères coprophages et l'abondance de ces insectes dans les crottes de différentes espèces de mammifères ont été abordées. L'étude a permis d'observer 226 individus de coléoptères coprophages Scarabeidae repartis en deux Sous-familles (Coprinae et Scarabeinae), quatre tribus (Aphodiini, Canthonini, Onthophagini et Sysiphini) et trois guildes fonctionnelles (rouleurs, fouisseurs et résidents).

La richesse taxonomique observée au cours de notre étude est relativement faible en comparaison aux résultats découlant d'autres travaux notamment ceux de Cambefort (1984), Krell et al. (2003) et Dosso et al. (2018). La différence s'expliquerait par des facteurs écologiques ou l'approche d'échantillonnage. En effet, la présence des insectes coprophages serait influencée par la saisonnalité (Cambefort, 1980), le régime alimentaire (Dosso, 2018), la structure de l'habitats (Cambefort, 1986). L'approche d'échantillonnage utilisé n'a pas nécessité un appât faisant intervenir l'humain. Les échantillons de crottes prélevés pourraient avoir été exposés pendant plusieurs jours aux conditions environnementales (humidité relative, température, luminosité...). Les crottes auraient perdu l'attrait pour les bousiers ou plusieurs individus ayant visité ces crottes pendant les premières heures d'expositions auraient quitté notamment les rouleurs. Ce qui expliquerait, d'ailleurs, la faible abondance relative de cette guilde dans l'ensemble des échantillons (4,42\% de Sysiphini et 2, 65\% de Canthonini). Par ailleurs, ces résultats sont conformes aux travaux de Cambefort (1984) qui rapporte que les Scarabaeidae rouleurs seraient en général absents en forêt et les fouisseurs en majorité les Onthophagini y seraient très abondants. Les fouisseurs (Onthophagini) représentent, dans cette étude, plus 66\% des individus. Les Aphodiini, les Coprini n'ont pas été observées au cours de cette étude mais elles pourraient être observées à Taï et dans d'autres milieux forestiers selon les travaux de Cambefort \& Bordat, (2003).

L'abondance relative des coléoptères coprophages diffère, en effet, en fonction des guildes et des Tribus. Toutefois, l'abondance des bousiers dans 
les crottes varient en fonction de l'espèce de mammifère responsable de la crotte. Ils seraient plus abondants dans les crottes de l'éléphant de forêt $(6,13$ individus/crotte) que dans celles récoltées chez d'autres Mammifères (buffle, potamochère, céphalophes). Le volume de crotte lié à la taille moyenne des individus de l'espèce de mammifère pourrait influencer le nombre de bousiers susceptible d'être attiré. Le buffle de forêt, les céphalophes et le potamochère qui sont comparativement plus petits que l'éléphant ont des crottes qui regorgeaient respectivement 4,71 individus/crotte, 3,22 individus/crotte, et 1,83 individus/crotte.

Cette étude préliminaire fournirait davantage d'informations sur la diversité des coléoptères coprophages, et les relations entre ces insectes et la faune mammalienne en augmentant aussi bien l'effort d'échantillonnage qu'en couvrant plusieurs saisons de l'année.

\section{Conclusion}

Au total, 226 individus de Coléoptères coprophages ont été récoltés au PNT, Sud-Ouest Côte d'Ivoire. Ces insectes sont repartis en deux Sousfamilles (Coprinae et Scarabeinae), quatre tribus (Aphodiini, Canthonini, Onthophagini et Sysiphini) et trois guildes fonctionnelles (rouleurs, fouisseurs et résidents). La Tribu des Onthophagini reste la plus abondante et représente $66,37 \%$ du total des individus observés. Une différence significative a été observée entre l'abondance moyenne de coléoptères coprophages dans les crottes l'éléphant de forêt et celle des crottes d'autres mammifères (potamochère, l'hippopotame nain et céphalophes). Cependant, aucune différence n'a été observée entre l'abondance de coléoptères coprophages dans les crottes de l'éléphant de forêt et dans celles du buffle de forêt. Cette étude qui présente un intérêt pour le suivi de la faune sauvage mérite d'être approfondie pour la gestion et la conservation de l'aire protégée.

\section{Remerciements}

Les auteurs voudraient exprimer leur reconnaissance à la Fondation Volkswagen pour le financement de cette étude. Ils expriment leurs sincères remerciements à l'OIPR (Office Ivoirien des Parcs et Réserves) pour avoir facilité la réalisation de ces travaux. Il convient de remercier également Monsieur Philippe Moretto de l'Association Catharsius (www.catharsius.fr) et Monsieur Kely Male Roger pour l'appui qu'ils ont apporté lors de l'étude. Nous remercions les référés anonymes dont les suggestions et commentaires ont permis d'améliorer la qualité du manuscript. 


\section{References:}

1. Bousquet, B. (1978). Un parc de forêt dense en Afrique. Le parc national de Taï (Côte d'Ivoire). Bois et forêts des tropiques, $179: 27$ 46.

2. Cambefort Y. (1980). Données préliminaires sur l'écologie des Scarabaeinae coprophages de Lamto. Annales de l'Université d'Abidjan. Série E Tome XII Ecologie, 60-79.

3. Cambefort Y. (1984). Nouveaux Scarabaeidae. str. de Côte-d'Ivoire et de pays : $326 \mathrm{p}$.

4. Cambefort Y. (1985). Les Coléoptères Scarabaeidae du Parc national de Taï (Côte d'lvoire). Revue Française d'Entomologie, 7: 337-342.

5. Cambefort Y. (1986). Rôle des coléoptères Scarabaeidae dans l'enfouissement des excréments en savane guinéenne de Côte-d'Ivoire. Acta Oecologica, Oecologia Generalis, 7 : 17-25.

6. Cambefort, Y. \& Borda, T. P. (2003). Coléoptères Scarabaeidae s. str., Aphodiidae et Ceratocanthidae du Mont Nimba et des régions limitrophes. In M. Lamotte et R. Roy (eds) : Le peuplement animal du Mont Nimba (Guinée, Côte d'Ivoire, Libéria). Mémoires du Muséum national d'Histoire naturelle, $190:$ 551-580.

7. Chatelain, C., Kadjo, B., Kone, I. \& Refisch, J. (2001). Relations Faune-Flore dans le Parc National de Taï: une étude bibliographique. Tropenbos-Côte d'Ivoire, $166 \mathrm{p}$.

8. Coleman, D.C. \& Hendrix, P. F., (2000). Invertebrates as Webmasters in Ecosystems, CABI Publishing, Wallingford, UK.

9. Couturier, G. \& Guillaumet, J. L. (1997). Les recherches entomologiques en forêt de Taï (Côte d'Ivoire). ORSTOM, 70-74.

10. Danon, A.S.D., Kra, K.D., Kwadjo, K.E, Douan, B.G, Loukou, K.K.S. \& Doumbia, M. (2017). Abondance et distribution des Coléoptères coprophages selon l'âge des plantations d'hévéa dans la localité de Toupah, au sud de la Côte d'Ivoire. Afrique Science 13: 421 - 434.

11. Davis, A.L.V., Frolov, A.V. \& Scholtz, C.H. 2008. The African Dung Beetle Genera. Protea Book House, Pretoria. $274 \mathrm{p}$

12. Dosso, K., Koffi, K. K.S \& Tiho, S. (2018). Etude préliminaire du peuplement de coléoptères bousiers inféodés aux excréments de buffles dans la réserve scientifique de Lamto. Revue de l'Environnement et de la Biodiversité-PASRES 3 : 44-53

13. Estes, J. A., Heithaus, M., McCauley, D. J., Rasher, D. B. \& Worm, B. (2016). Megafaunal impacts on structure and function of ocean ecosystems. Annual Review of Environment and Resources, 41: 83116.

14. Favila, M. \& Halffter, G. (1997). Indicator groups for measuring biodiversity, Acta Zoologica Mexicana, 72: 1-25. 
15. Hoppe-Dominik, B., Kühl, H. S., Radl, G. \& Fischer, F. (2011). Longterm monitoring of large rainforest mammals in the Biosphere Reserve of Taï National Park, Côte d'Ivoire. African Journal of Ecology, 49(4) :450-458.

16. Kolongo, T. S. D, Decocq, G., Adou, Y. C. Y., Blom, E. C. \& Rompaey, R. S. A. (2006). Plant species diversity in the southern part of the Tai National Park (Côte d'Ivoire). Biodiversity and Conservation, 15 : 2123-2142.

17. Kouakou, Y. C. (2004). Influence des perturbations anthropiques sur les coléoptères coprophages : cas du pâturage et de l'urbanisation respectivement en région de savane (Toumodi) et de forêt (Abidjan). Diplôme d'Etudes Approfondies à l'Université d'Abobo-Adjamé, Abidjan, Côte d'Ivoire, UFR-SN, 66 pages.

18. Krell, F.-T., Krell-Westerwalbesloh, S., Weiss, I., Eggleton, P. \& Linsenmair,K. E. (2003). Spatial separation of Afrotropical dung beetle guilds: a trade-off between competitive superiority and energetic constraints (Coleoptera: Scarabaeidae), Ecography, 26: 210222

19. Krell-Westerwalbesloh, S., Krell, F. T. \& Linsenmair, K. E. (2004)Diel separation of Afrotropical dung beetle and neglecting resources (Coleoptera: Scarabaeoidea). Journal of Natural History, 38: 22252249.

20. Myers, M., Mittermeier, R. A., Mittermeier, C. G., Da Fonseca, G. A. B., \& Kent, J. (2000). Biodiversity hot spots for conservation priorities. Nature, 403: 853-858.

21. Nichols, E., Spector, S., Louzada, J., Larsen, T., Amezquita, S. \& Favila, M. E. (2008). Ecological functions and ecosystem services provided by Scarabaeinae dung beetles. Biological Conservation 141(6): 1461-1474.

22. OIPR-Office Ivoirien des Parcs et Réserves (2014). Plan d'aménagement et de gestion du parc national de Taï 2014-2018, Abidjan (Côte d'Ivoire), $131 \mathrm{p}$.

23. Riezebos, E. P., Vooren, A. P. \& Guillaumet, J. L. (1994). Le Parc National de Taï, Cote d'Ivoire. I: Synthèse des connaissances. II: Bibliographie. Tropenbos Serie 8, Wageningen. 322 pp

24. Ripple, W. J., Abernethy, K., Betts, M. G., Chapron, G., Dirzo, R., Galetti, M., Levi, T., Lindsey, P. A., MacDonald, D. W., Machovina, B., Newsome, T. M., Peres, C.A., Wallache, A. D., Wolf, C., Young, H. (2016). Bushmeat hunting and extinction risk to the world's mammals. Royal Society Open Science, 3, 160498 
25. Samways, M. J. (1993). Insects in biodiversity conservation: some perspectives and directives, Biodiversity and Conservation, 2: 258282.

26. Simon, A. (2010). Méthodes de recherche des coléoptères coprophages : retour d'expérience. Invertébrés Amoricains 6: 34-44.

27. White, L. \& Edwards, A. (2000). Conservation Research in the African Rain Forests: A Technical Handbook. Wildlife Conservation Society, New. York. 444 pp.

28. Zaime A. \& Gautier J. Y. (1989). Comparaison des régimes alimentaires de trois espèces sympatriques de Gerbillidae en milieu Saharien au Maroc. Revue d'Ecologie, Terre et vie 44 : 263-278.

29. Zinga K.C.R., Mbang, N.O., Mounioko, F., Mounioko, F., Mavoungou, J.F., M'batchi, B. (2016). Repartition des Glossines dans la Province de l'ogooue Ivindo Ancien foyer de Trypanosomose Humaine Africaine. European Scientific Journal 12 : 281-297.

30. Zinque M. H. (2012). Le rôle écologique des Scarabaeidae coprophages dans le destin des graines de Uapaca spp. disséminées par le gorille des plaines de l'Ouest (Gorilla gorilla gorilla) en forêt dense camerounaise. Travail de fin d'études : Gembloux Agro-Bio Tech, Université de Liège. 85 pp. + Annexes. 